\title{
A New Numerical Algorithm for Two-Point Boundary Value Problems
}

\author{
Lihua Guo, Boying Wu, and Dazhi Zhang \\ Department of Mathematics, Harbin Institute of Technology, Harbin 150001, China \\ Correspondence should be addressed to Dazhi Zhang; zhang-d-z@163.com
}

Received 8 April 2014; Revised 17 June 2014; Accepted 17 June 2014; Published 30 June 2014

Academic Editor: Jisheng Kou

Copyright ( 2014 Lihua Guo et al. This is an open access article distributed under the Creative Commons Attribution License, which permits unrestricted use, distribution, and reproduction in any medium, provided the original work is properly cited.

We present a new numerical algorithm for two-point boundary value problems. We first present the exact solution in the form of series and then prove that the $n$-term numerical solution converges uniformly to the exact solution. Furthermore, we establish the numerical stability and error analysis. The numerical results show the effectiveness of the proposed algorithm.

\section{Introduction}

It is well known that many problems can be presented by the following two-point boundary value problems:

$$
\begin{gathered}
{\left[x^{\alpha} y^{\prime}(x)\right]^{\prime}=f(x, y), \quad x \in(0,1),} \\
y(0)=a, \quad y(1)=b,
\end{gathered}
$$

where $\alpha \in[0,1], a$, and $b$ are finite constants.

Problem (1) arises from many fields of applied mathematics and physics, such as nuclear physics, economical system, chemical engineering, and underground water flow. Therefore, this problem has attracted considerable attention. For example, Aziz and Kumar [1,2] presented a finite difference method based on nonuniform mesh to solve this problem. Kumar [3, 4] presented a second order spline finite difference method to solve (1) by using a spline function. Rashidinia et al. [5] presented a parametric spline method for (1). For these references, please see [6-10].

In this paper, we propose a new numerical algorithm to solve (1) by using the reproducing kernel theory. By homogenizing the boundary value conditions, (1) is converted into a nonlinear operator equation. We show that the solution of (1) is equivalent to the solution of the operator equation, and its exact solution $y(x)$ can be represented in the form of series. Furthermore, we prove that the $n$-term numerical solution $y_{n}(x)$ converges uniformly to the exact solution.
Then, numerical stability and error analysis of the method are presented. Numerical results show that this method has high accuracy.

The paper is organized as follows. In Section 2, fundamental definitions and theorems of the reproducing kernel theory are given. In Section 3, the nonlinear operator equation is constructed. The new numerical algorithm is presented in Section 4. In Section 5, we apply our method to linear and nonlinear numerical examples and illustrate the applicability of the presented method. Section 6 ends this paper with a brief conclusion.

\section{Fundamental Definitions and Theorems}

In this section, we show some fundamental theories of the reproducing kernel space $[11,12]$.

Definition 1. Let $H$ denote a Hilbert space, which is composed of functions defined on an abstract set $D$ and admits $a$ reproducing kernel $K(x, y)$. That is, for each fixed $y \in D$, $K(x, y)$ belongs to $H$ as a function in $x$ and for any $f \in H$,

$$
\langle f(x), K(x, y)\rangle_{H}=f(y) .
$$

We call (2) the reproducing property of $K(x, y)$. 
Theorem 2. Let $H$ be a Hilbert space; let $\left\{\varphi_{i}(x)\right\}_{i=1}^{\infty}$ be a complete function system; that is,

$$
\left\langle\varphi_{i}(x), \varphi_{j}(x)\right\rangle_{H}= \begin{cases}0, & i \neq j, \\ 1, & i=j\end{cases}
$$

then $K(x, y)=\sum_{i=1}^{\infty} \varphi_{i}(x) \bar{\varphi}_{i}(y)$ is the reproducing kernel of $H$.

Proof. In fact, $\forall f(x) \in H ; f(x)=\sum_{i=1}^{\infty} a_{i} \varphi_{i}(x), a_{i} \in \mathbb{C}$. In view of (2) and (3), we have

$$
\begin{aligned}
\langle f(x), K(x, y)\rangle_{H} & =\left\langle\sum_{i=1}^{\infty} a_{i} \varphi_{i}(x), \sum_{i=1}^{\infty} \varphi_{i}(x) \bar{\varphi}_{i}(y)\right\rangle_{H} \\
& =\sum_{i=1}^{\infty} a_{i} \varphi_{i}(y)=f(y) .
\end{aligned}
$$

Definition 3. The reproducing kernel space $W_{2}^{m}[0,1]$ is defined as follows.

$W_{2}^{m}[0,1]=\left\{f(x) \mid f^{(m-1)}(x)\right.$ is an absolutely continuous function, $\left.f^{(m)}(x) \in L^{2}[0,1], x \in[0,1]\right\}$.

The inner product and norm are defined as, respectively, $\forall f(x), g(x) \in W_{2}^{m}[0,1]$,

$$
\begin{gathered}
\langle f(x), g(x)\rangle_{W_{2}^{m}}=\sum_{i=0}^{m-1} f^{i}(0) g^{i}(0)+\int_{0}^{1} f^{m}(x) g^{m}(x) d x, \\
\|f(x)\|=\sqrt{\langle f(x), f(x)\rangle_{W_{2}^{m}}}
\end{gathered}
$$

Theorem 4. $W_{2}^{m}[0,1]$ is a complete space with respect to $\|\cdot\|_{W_{2}^{m}}$.

Proof. If the reproducing kernel $K(x, y)$ of the space $W_{2}^{m}[0,1]$ exists, in view of (2) and Cauchy-Schwartz's inequality, we have

$$
\begin{aligned}
f(y) & =\langle f(x), K(x, y)\rangle_{W_{2}^{m}} \leq\|f\|_{W_{2}^{m}}\|K(x, y)\|_{W_{2}^{m}} \\
& =\|f\|_{W_{2}^{m}} \sqrt{\langle K(x, y), K(x, y)\rangle_{W_{2}^{m}}} \\
& =\|f\|_{W_{2}^{m}} \sqrt{K(y, y)}
\end{aligned}
$$

which shows that $f(y)$ is a bounded linear function on $W_{2}^{m}[0,1]$. Hence, there exists a Cauchy sequence $\left\{f_{n}(x)\right\} \in$ $W_{2}^{m}[0,1]$. By (2) and Cauchy-Schwartz's inequality, we obtain

$$
\begin{aligned}
\| f_{n}(y) & -f_{m}(y) \| \\
= & \left\|\left\langle f_{n}(x)-f_{m}(x), K(x, y)\right\rangle_{W_{2}^{m}}\right\| \\
\leq & \left\|f_{n}(x)-f_{m}(x)\right\|_{W_{2}^{m}}\|K(x, y)\|_{W_{2}^{m}} \\
= & \left\|f_{n}(x)-f_{m}(x)\right\|_{W_{2}^{m}} \sqrt{\langle K(x, y), K(x, y)\rangle_{W_{2}^{m}}} \\
\leq & \left\|f_{n}(x)-f_{m}(x)\right\|_{W_{2}^{m}} \sqrt{K(y, y) .}
\end{aligned}
$$

Therefore, there exists $f(x) \in W_{2}^{m}[0,1]$ such that $\lim _{n \rightarrow \infty} f_{n}(x)=f(x)$. Furthermore, we have $\lim _{n \rightarrow \infty}\left\|f_{n}\right\|_{W_{2}^{m}}=\|f\|_{W_{2}^{m}}$ and $\lim _{n \rightarrow \infty}\left\langle f_{n}, g_{n}\right\rangle_{W_{2}^{m}}=$ $\langle f, g\rangle_{W_{2}^{m}}$. So the proof of Theorem 4 is complete.

\section{Structure of the Nonlinear Operator}

Now, we show the method to solve (1). By transformation, we have

$$
x^{\alpha} y^{\prime \prime}(x)+\alpha x^{\alpha-1} y^{\prime}(x)=\left[x^{\alpha} y^{\prime}(x)\right]^{\prime}=f(x, y) .
$$

That is,

$$
\begin{gathered}
y^{\prime \prime}(x)+\alpha x^{-1} y^{\prime}(x)=x^{-\alpha} f(x, y), \\
y^{\prime \prime}(x)+a(x) y^{\prime}(x)=g(x, y),
\end{gathered}
$$

where $a(x)=\alpha x^{-1}$ and $g(x, y)=x^{-\alpha} f(x, y)$. Let

$$
L y=y^{\prime \prime}+a(x) y^{\prime}, \quad 0<x<1,
$$

with $L: W_{2}^{3}[0,1] \rightarrow W_{2}^{1}[0,1]$. By homogenizing the boundary value conditions, (1) can be converted into the equivalent nonlinear operator equation:

$$
\begin{gathered}
L y=g(x, y), \quad 0<x<1, \\
y(0)=y(1)=0 .
\end{gathered}
$$

For any $y(x) \in W_{2}^{3}[0,1]$ and each fixed point $x \in[0,1]$,

$$
\varphi_{i}(x)=K\left(x_{i}, y\right), \quad \psi_{i}(x)=L^{*} \varphi_{i}(x),
$$

where $\left\{x_{i}\right\}_{i=1}^{\infty}$ is a different dense point set on $[0,1], L^{*}$ is the conjugate operator of $L$, and $K(x, y)$ is the reproducing kernel of $W_{2}^{3}[0,1]$. In terms of the property of (2) and the inner product, we obtain

$$
\begin{aligned}
\left\langle y(x), \psi_{i}(x)\right\rangle_{W_{2}^{3}} & =\left\langle y(x), L^{*} \varphi_{i}(x)\right\rangle_{W_{2}^{3}} \\
& =\left\langle L y(x), \varphi_{i}(x)\right\rangle_{W_{2}^{1}} \\
& =\left\langle L y(x), K\left(x_{i}, y\right)\right\rangle_{W_{2}^{1}} \\
& =L y\left(x_{i}\right), \quad i=1,2, \ldots
\end{aligned}
$$

Therefore, we can see that the solution of (1) is equivalent to the solution of (11).

\section{Solving the Problem}

Through the normal orthogonal process, $\left\{\bar{\psi}_{i}(x)\right\}_{i=1}^{\infty}$ of $W_{2}^{m}[0,1]$ can be derived from $\left\{\psi_{i}(x)\right\}_{i=1}^{\infty}$. That is,

$$
\bar{\psi}_{i}(x)=\sum_{k=1}^{i} \beta_{i k} \psi_{k}(x),
$$

where $\beta_{i k}$ are the orthogonalization coefficients. 
Theorem 5. If $\left\{x_{i}\right\}_{i=1}^{\infty}$ is the different dense point set on $[0,1]$ and $y(x)$ is the exact solution of (11) in $W_{2}^{3}[0,1]$, then

$$
y(x)=\sum_{i=1}^{\infty} \sum_{k=1}^{i} \bar{\beta}_{i k} g\left(x_{k}, y\left(x_{k}\right)\right) \bar{\psi}_{i}(x) .
$$

Proof. Since $y(x) \in W_{2}^{3}[0,1]$ and $\left\{\bar{\psi}_{i}(x)\right\}_{i=1}^{\infty}$ is a normal complete orthogonal system, then $y(x)$ can be expanded by Fourier series with the normal orthogonal basis; namely,

$$
y(x)=\sum_{i=1}^{\infty}\left\langle y(x), \bar{\psi}_{i}(x)\right\rangle \bar{\psi}_{i}(x)
$$

Because $W_{2}^{3}[0,1]$ is complete, $y(x)$ is uniformly convergent in the sense of $\|\cdot\|_{W_{2}^{3}}$. Note that $y(x) \in W_{2}^{3}[0,1] ; y(x)$ is absolutely continuous, in terms of (2) and (14); we obtain

$$
\begin{aligned}
y(x) & =\sum_{i=1}^{\infty}\left\langle y(x), \bar{\psi}_{i}(x)\right\rangle \bar{\psi}_{i}(x) \\
& =\sum_{i=1}^{\infty}\left\langle y(x), \sum_{k=1}^{i} \beta_{i k} \psi_{k}(x)\right\rangle \bar{\psi}_{i}(x) \\
& =\sum_{i=1}^{\infty} \sum_{k=1}^{i} \bar{\beta}_{i k}\left\langle y(x), \psi_{k}(x)\right\rangle \bar{\psi}_{i}(x) \\
& =\sum_{i=1}^{\infty} \sum_{k=1}^{i} \bar{\beta}_{i k}\left\langle y(x), L^{*} \varphi_{k}(x)\right\rangle \bar{\psi}_{i}(x) \\
& =\sum_{i=1}^{\infty} \sum_{k=1}^{i} \bar{\beta}_{i k}\left\langle L y(x), \varphi_{k}(x)\right\rangle \bar{\psi}_{i}(x) \\
& =\sum_{i=1}^{\infty} \sum_{k=1}^{i} \bar{\beta}_{i k}\left\langle L y(x), K\left(x_{k}, y\right)\right\rangle \bar{\psi}_{i}(x) \\
& =\sum_{i=1}^{\infty} \sum_{k=1}^{i} \bar{\beta}_{i k} L y\left(x_{k}\right) \bar{\psi}_{i}(x) \\
& =\sum_{i=1}^{\infty} \sum_{k=1}^{i} \bar{\beta}_{i k} g\left(x_{k}, y\left(x_{k}\right)\right) \bar{\psi}_{i}(x) .
\end{aligned}
$$

The proof is complete.

By truncating the right hand of (15), we obtain the approximate solution of (11); namely,

$$
y_{n}(x)=\sum_{i=1}^{n} \sum_{k=1}^{i} \bar{\beta}_{i k} g\left(x_{k}, y\left(x_{k}\right)\right) \bar{\psi}_{i}(x)
$$

where $y_{n}(x)$ is the $n$-term intercept of $y(x)$ in (15). In view of the completeness of the reproducing kernel space, $y_{n}(x) \rightarrow$ $y(x)$ as $n \rightarrow \infty$.
Next, in order to discuss the uniform convergence of the approximate solution, for any fixed $y_{0}(x) \in W_{2}^{3}[0,1], y_{n}(x)=$ $\sum_{i=1}^{n} a_{i} \bar{\psi}_{i}(x)$ with $a_{i}=\sum_{k=1}^{i} \bar{\beta}_{i k} g\left(x_{k}, y\left(x_{k}\right)\right)$; we construct an iterative sequence $\left\{y_{n}(x)\right\}$. That is,

$$
\begin{aligned}
a_{1} & =\bar{\beta}_{11} g\left(x_{1}, y_{0}\left(x_{1}\right)\right), \\
a_{2}= & \sum_{k=1}^{2} \bar{\beta}_{2 k} g\left(x_{k}, y_{k-1}\left(x_{k}\right)\right) \\
& =\bar{\beta}_{21} g\left(x_{1}, y_{0}\left(x_{1}\right)\right)+\bar{\beta}_{22} g\left(x_{2}, y_{1}\left(x_{2}\right)\right), \\
\vdots & \\
a_{n}= & \sum_{k=1}^{n} \bar{\beta}_{n k} g\left(x_{k}, y_{n-1}\left(x_{k}\right)\right) .
\end{aligned}
$$

Theorem 6. Assume the following.

(a) $\left\{x_{i}\right\}_{i=1}^{\infty}$ is a different dense point set on $[0,1]$.

(b) $\left\{\bar{\psi}_{i}(x)\right\}_{i=1}^{\infty}$ is a normal orthogonal system.

(c) $\left\|y_{n}(x)\right\|_{W_{2}^{3}}$ is bounded.

(d) $g(x, y(x)) \in W_{2}^{1}[0,1]$, for any $y(x) \in W_{2}^{3}[0,1], x \in$ $[0,1]$.

Then the iterative formula $y_{n}(x)$ converges uniformly to the exact solution $y(x)$ of (11).

Proof. By $y_{n}(x)=\sum_{i=1}^{n} a_{i} \bar{\psi}_{i}(x)$, we have $y_{n+1}(x)=y_{n}(x)+$ $a_{n+1} \bar{\psi}_{n+1}(x)$. In view of the orthonormality of $\left\{\bar{\psi}_{i}(x)\right\}_{i=1}^{\infty}$, we have

$$
\begin{aligned}
\left\|y_{n+1}(x)\right\|^{2} & =\left\|y_{n}(x)+a_{n+1} \bar{\psi}_{n+1}(x)\right\|^{2} \\
& =\left\|y_{n}(x)\right\|^{2}+\left\|a_{n+1} \bar{\psi}_{n+1}(x)\right\|^{2} \\
& =\left\|y_{n}(x)\right\|^{2}+a_{n+1}^{2} \\
& =\left\|y_{n-1}(x)\right\|^{2}+a_{n}^{2}+a_{n+1}^{2} \\
& \vdots \\
& =a_{1}^{2}+\cdots+a_{n}^{2}+a_{n+1}^{2}=\sum_{i=1}^{n+1} a_{i}^{2} .
\end{aligned}
$$

In view of the boundedness of $\left\|y_{n}(x)\right\|_{W_{2}^{3}}$, we have $\sum_{i=1}^{\infty} a_{i}^{2}<$ $\infty$. Let $n>m$ and $m, n \rightarrow \infty$, owing to $\left(y_{n}-y_{n-1}\right) \perp\left(y_{n-1}-\right.$ $\left.y_{n-2}\right) \perp \cdots \perp\left(y_{m+1}-y_{m}\right)$; we obtain

$$
\begin{aligned}
\| y_{n} & -y_{m} \|^{2} \\
& =\left\|y_{n}-y_{n-1}+y_{n-1}-y_{n-2}+\cdots+y_{m+1}-y_{m}\right\|^{2} \\
& =\left\|y_{n}-y_{n-1}\right\|^{2}+\left\|y_{n-1}-y_{n-2}\right\|^{2}+\cdots+\left\|y_{m+1}-y_{m}\right\|^{2} \\
& =\left\|a_{n} \bar{\psi}_{n}(x)\right\|^{2}+\left\|a_{n-1} \bar{\psi}_{n-1}(x)\right\|^{2}+\cdots+\left\|a_{m+1} \bar{\psi}_{m+1}(x)\right\|^{2} \\
& =a_{n}^{2}+a_{n-1}^{2}+\cdots+a_{m+1}^{2}=\sum_{i=m+1}^{n} a_{i}^{2} .
\end{aligned}
$$



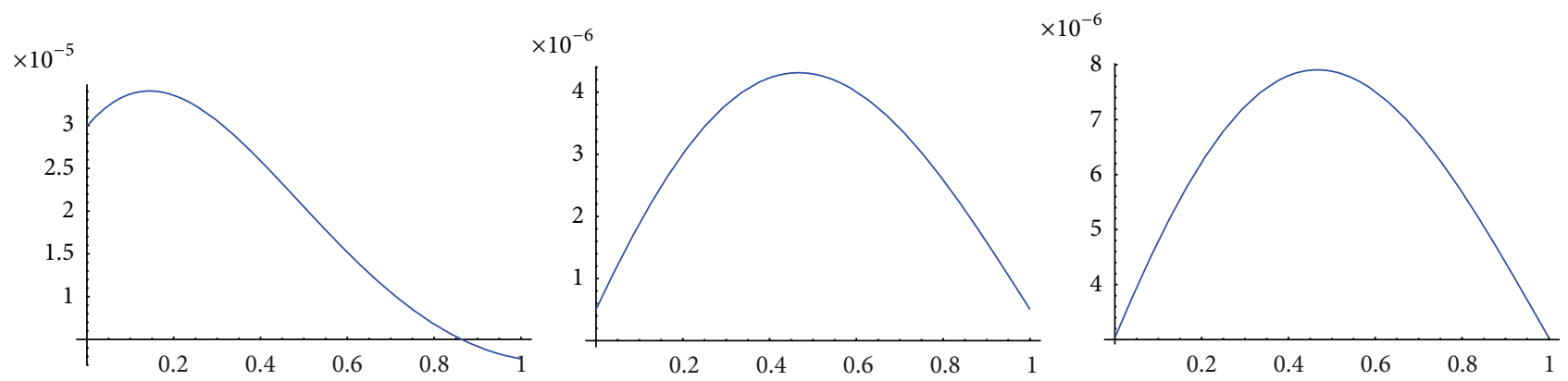

FIGURE 1: R.M.S of the exact solution $y^{k}(x)$ and the approximate solution $y_{n}^{k}(x)(k=0,1,2)$.

By the completeness of $W_{2}^{3}[0,1]$, there exists $y(x) \in W_{2}^{3}[0,1]$, such that $y_{n}(x) \rightarrow y(x)$ in the sense of $\|\cdot\|_{W_{2}^{3}}$ as $n \rightarrow \infty$. The proof is complete.

Theorem 7. Error Analysis. Assume that the conditions of Theorem 6 are satisfied; then the error of the numerical solution is monotonically decreasing with the increasing of nodes; that is, $\varepsilon_{n}(x) \rightarrow 0$, as $n \rightarrow \infty$.

Proof. It is easy to see that

$$
\begin{aligned}
\left\|\varepsilon_{n}(x)\right\| & =\left\|y(x)-y_{n-1}(x)\right\| \\
& =\left\|\sum_{i=1}^{\infty} a_{i} \bar{\psi}_{i}(x)-\sum_{i=1}^{n-1} a_{i} \bar{\psi}_{i}(x)\right\| \\
& =\left\|\sum_{i=n}^{\infty} a_{i} \bar{\psi}_{i}(x)\right\| \geq\left\|\sum_{i=n+1}^{\infty} a_{i} \bar{\psi}_{i}(x)\right\| \\
& =\left\|y(x)-y_{n}(x)\right\|=\left\|\varepsilon_{n+1}(x)\right\| .
\end{aligned}
$$

Thus we complete the proof.

Now, we show the stability of the proposed method.

Theorem 8. For (11), if $g(x, y)$ has a small perturbation $\delta$, then the proposed method is stable.

Proof. For the problem (11), if $g(x, y)$ has a small perturbation $\delta$, then $L y=g(x, y)+\delta$. Let $\widetilde{y}_{n}(x)$ be the numerical solution of $L \tilde{y}=g(x, y)+\delta$; in view of Theorems 5 and 6 , we have

$$
\begin{aligned}
& y_{n}(x)=\sum_{i=1}^{n} \sum_{k=1}^{i} \bar{\beta}_{i k} g\left(x_{k}, y\left(x_{k}\right)\right) \bar{\psi}_{i}(x), \\
& \tilde{y}_{n}(x)=\sum_{i=1}^{n} \sum_{k=1}^{i} \bar{\beta}_{i k}\left[g\left(x_{k}, y\left(x_{k}\right)\right)+\delta\right] \bar{\psi}_{i}(x) .
\end{aligned}
$$

Hence,

$$
\left\|y_{n}(x)-\tilde{y}_{n}(x)\right\|=|\delta| \cdot\left\|\sum_{i=1}^{n} \sum_{k=1}^{i} \bar{\beta}_{i k} \bar{\psi}_{i}(x)\right\| \leq|\delta| \cdot M<\varepsilon .
$$

TABle 1: The M.A. error of Example 1.

\begin{tabular}{lcccc}
\hline$N$ & Method in [13] & Method in [2] & Method in [5] & Our method \\
\hline 16 & $1.15(-2)$ & $2.10(-2)$ & $7.64(-4)$ & $9.91(-5)$ \\
32 & $2.90(-3)$ & $5.20(-3)$ & $2.15(-4)$ & $1.74(-5)$ \\
64 & $7.28(-4)$ & $1.30(-3)$ & $5.55(-5)$ & $5.78(-6)$ \\
128 & $1.82(-4)$ & $3.30(-4)$ & $1.39(-5)$ & $1.51(-6)$ \\
256 & - & - & - & $3.55(-7)$ \\
\hline
\end{tabular}

That is, $\forall \varepsilon>0, \exists \delta=\varepsilon / M$, such that

$$
\left\|y_{n}(x)-\tilde{y}_{n}(x)\right\|<\varepsilon .
$$

Therefore, the method is stable.

\section{Numerical Examples}

5.1. Example 1. Consider the following linear two-point boundary value problem [5]:

$$
\begin{gathered}
x^{-\alpha}\left(x^{\alpha} y^{\prime}\right)^{\prime}=\beta x^{\beta-2}\left(\alpha+\beta-1+\beta x^{\beta}\right) y, \\
y(0)=1, \quad y(1)=e .
\end{gathered}
$$

The exact solution is $y(x)=\exp \left(x^{\beta}\right)$ with $\alpha=0.5$ and $\beta=4$. The maximum absolute errors (M.A. error) are tabulated in Table 1; the Root-mean-square errors (R.M.S) of the exact solution $y^{k}(x)$ and the approximate solution $y_{n}^{k}(x)(k=$ $0,1,2)$ are shown in Figure 1. From the numerical results, we can see that the present method produces better approximate solution than [5] and the error of the numerical solution is monotonically decreasing with the increase of nodes.

5.2. Example 2. Consider the nonlinear singular two-point boundary value problem $[14,15]$ :

$$
\begin{aligned}
& y^{\prime \prime}+\frac{1}{2 x} y^{\prime}=e^{y}\left(\frac{1}{2}-e^{y}\right), \\
& y(0)=\ln 2, \quad y(1)=0 .
\end{aligned}
$$

The exact solution is $y(x)=\ln \left(2 /\left(1+x^{2}\right)\right)$. When $N=8,16,32,64,128,256$, the maximum absolute errors 

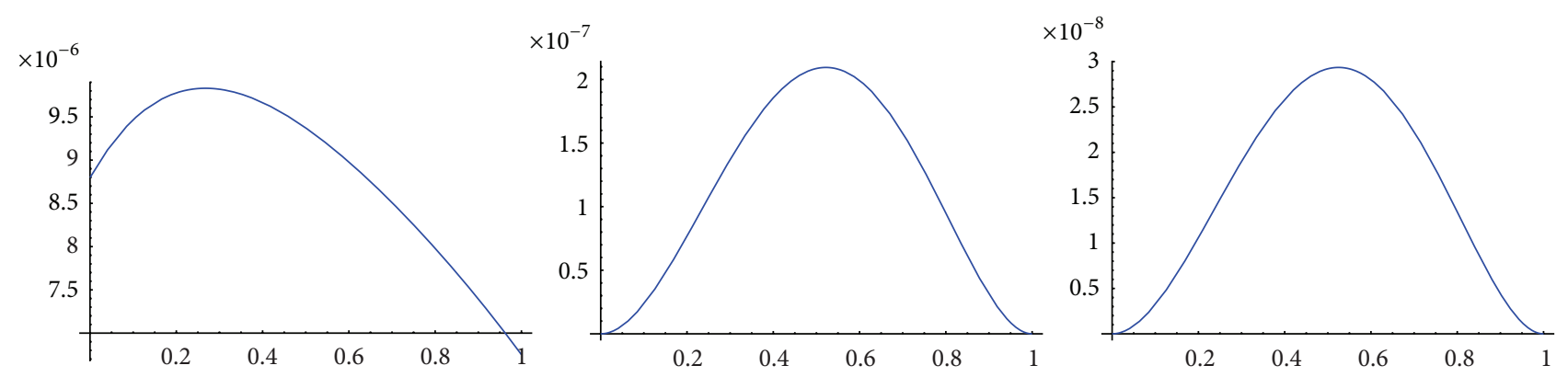

FIGURE 2: R.M.S of the exact solution $y^{k}(x)$ and the approximate solution $y_{n}^{k}(x)(k=0,1,2)$.

TABLE 2: The M.A. error in solutions of Example 2.

\begin{tabular}{lcccc}
\hline$x$ & $\mid y(x)-$ Padé $[5 / 5](x) \mid$ method in $[16]$ & $\left|y(x)-\phi_{10}(x)\right|$ method in $[14,15]$ & $N$ & Our method \\
\hline $10^{-1}$ & $4.432 E-04$ & $1.734 E-17$ & $3.956(-6)$ \\
$10^{-2}$ & $1.368 E-04$ & $0.000 E-00$ & 16 & $9.822(-7)$ \\
$10^{-3}$ & $1.300 E-05$ & $1.602 E-17$ & 32 & $1.244(-7)$ \\
$10^{-4}$ & $1.219 E-06$ & $1.077 E-17$ & 64 & $6.325(-8)$ \\
$10^{-5}$ & $2.659 E-06$ & $8.279 E-18$ & 128 & $2.333(-8)$ \\
$10^{-6}$ & $2.803 E-06$ & $2.212 E-17$ & 256 \\
\hline
\end{tabular}

(M.A. error) are tabulated in Table 2; the Root-mean-square error (R.M.S) of the exact solution $y^{k}(x)$ and the approximate solution $y_{n}^{k}(x)(k=0,1,2)$ are shown in Figure 2 . From the numerical results, we can see that the error of the numerical solution is monotonically decreasing with the increase of nodes.

\section{Conclusions}

In this paper, we use the reproducing kernel iterative method to solve a class of two-point boundary value problems. By homogenizing the boundary conditions, the two-point boundary value problem is converted into the equivalent nonlinear operator equation. We prove that their solutions are equivalent, the exact solution $y(x)$ can be represented in the form of series, and the $n$-term numerical solution $y_{n}(x)$ converges uniformly to the exact solution $y(x)$. Furthermore, we show the analysis of error and stability for the method. At last, numerical results show the high accuracy and the validity of this method.

\section{Conflict of Interests}

The authors declare that there is no conflict of interests regarding the publication of this paper.

\section{Acknowledgments}

This work is supported by the National Science Foundation of China (11271100, 11301113, and 71303067), Harbin Science and Technology Innovative Talents Project of Special Fund (2013RFXYJ044), China Postdoctoral Science Foundation Fund Project (Grant no. 2013M541400), the Heilongjiang
Postdoctoral Fund (Grant no. LBH-Z12102), and the Fundamental Research Funds for the Central Universities (Grant no. HIT.HSS.201201).

\section{References}

[1] T. Aziz and M. Kumar, "A fourth-order finite-difference method based on non-uniform mesh for a class of singular twopoint boundary value problems," Journal of Computational and Applied Mathematics, vol. 136, no. 1-2, pp. 337-342, 2001.

[2] M. Kumar and T. Aziz, "A non-uniform mesh finite difference method and its convergence for a class of singular two-point boundary value problems," International Journal of Computer Mathematics, vol. 81, no. 12, pp. 1507-1512, 2004.

[3] M. Kumar, "A second order spline finite difference method for singular two-point boundary value problems," Applied Mathematics and Computation, vol. 142, no. 2-3, pp. 283-290, 2003.

[4] M. Kumar, "Higher order method for singular boundary-value problems by using spline function," Applied Mathematics and Computation, vol. 192, no. 1, pp. 175-179, 2007.

[5] J. Rashidinia, Z. Mahmoodi, and M. Ghasemi, "Parametric spline method for a class of singular two-point boundary value problems," Applied Mathematics and Computation, vol. 188, no. 1, pp. 58-63, 2007.

[6] H. Yao and Y. Lin, "Solving singular boundary-value problems of higher even-order," Journal of Computational and Applied Mathematics, vol. 223, no. 2, pp. 703-713, 2009.

[7] Y. Lin, J. Niu, and M. Cui, "A numerical solution to nonlinear second order three-point boundary value problems in the reproducing kernel space," Applied Mathematics and Computation, vol. 218, no. 14, pp. 7362-7368, 2012.

[8] J. Niu, Y. Z. Lin, and C. P. Zhang, "Approximate solution of nonlinear multi-point boundary value problem on the halfline," Mathematical Modelling and Analysis, vol. 17, no. 2, pp. 190-202, 2012. 
[9] C. Song, J. Li, and R. Gao, "Nonexistence of global solutions to the initial boundary value problem for the singularly perturbed sixth-order boussinesq -type equation," Journal of Applied Mathematics, vol. 2014, Article ID 928148, 7 pages, 2014.

[10] B. Orel and A. Perne, "Chebyshev-fourier spectral methods for nonperiodic boundary value problems," Journal of Applied Mathematics, vol. 2014, Article ID 572694, 10 pages, 2014.

[11] N. Aronszajn, “Theory of reproducing kernels," Transactions of the American Mathematical Society, vol. 68, pp. 337-404, 1950.

[12] M. G. Cui and Y. Z. Lin, Nonlinear Numerical Analysis in the Reproducing Kernel, Nova Science, New York, NY, USA, 2009.

[13] R. K. Pandy and Singh. A. K., "On the convergence of secondorder finite difference method for weakly regular singular boundary value problems," International Journal of Computer Mathematics, vol. 85, no. 12, pp. 1807-1814, 2008.

[14] A. E. Ebaid, "Exact solutions for a class of nonlinear singular two-point boundary value problems: the decomposition method," Zeitschrift fur Naturforschung A: Journal of Physical Sciences, vol. 65, no. 3, pp. 145-150, 2010.

[15] A. Ebaid, "A new analytical and numerical treatment for singular two-point boundary value problems via the Adomian decomposition method," Journal of Computational and Applied Mathematics, vol. 235, no. 8, pp. 1914-1924, 2011.

[16] S. M. El-Sayed, "Integral methods for computing solutions of a class of singular two-point boundary value problems," Applied Mathematics and Computation, vol. 130, no. 2-3, pp. 235-241, 2002. 


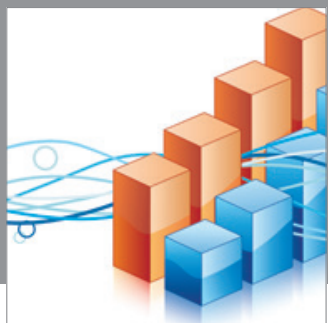

Advances in

Operations Research

mansans

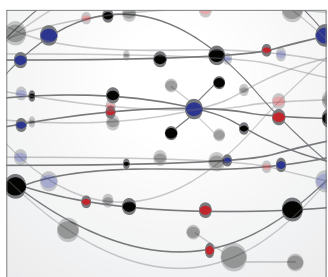

The Scientific World Journal
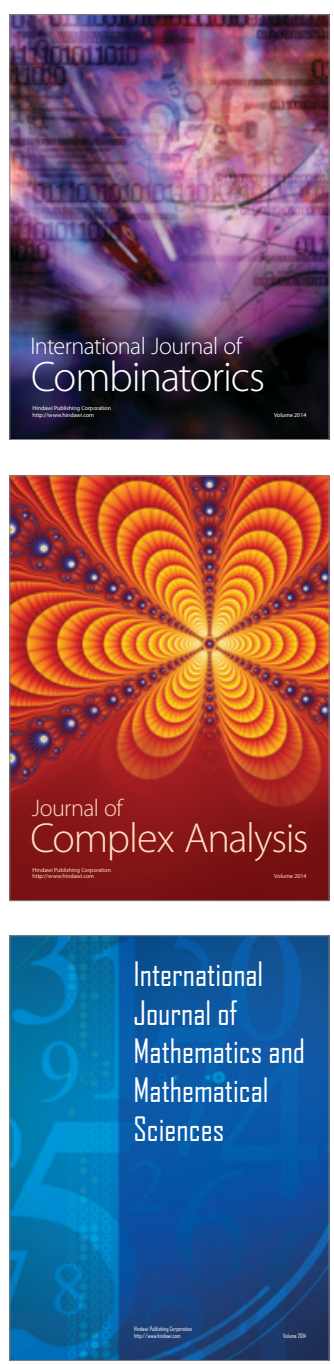
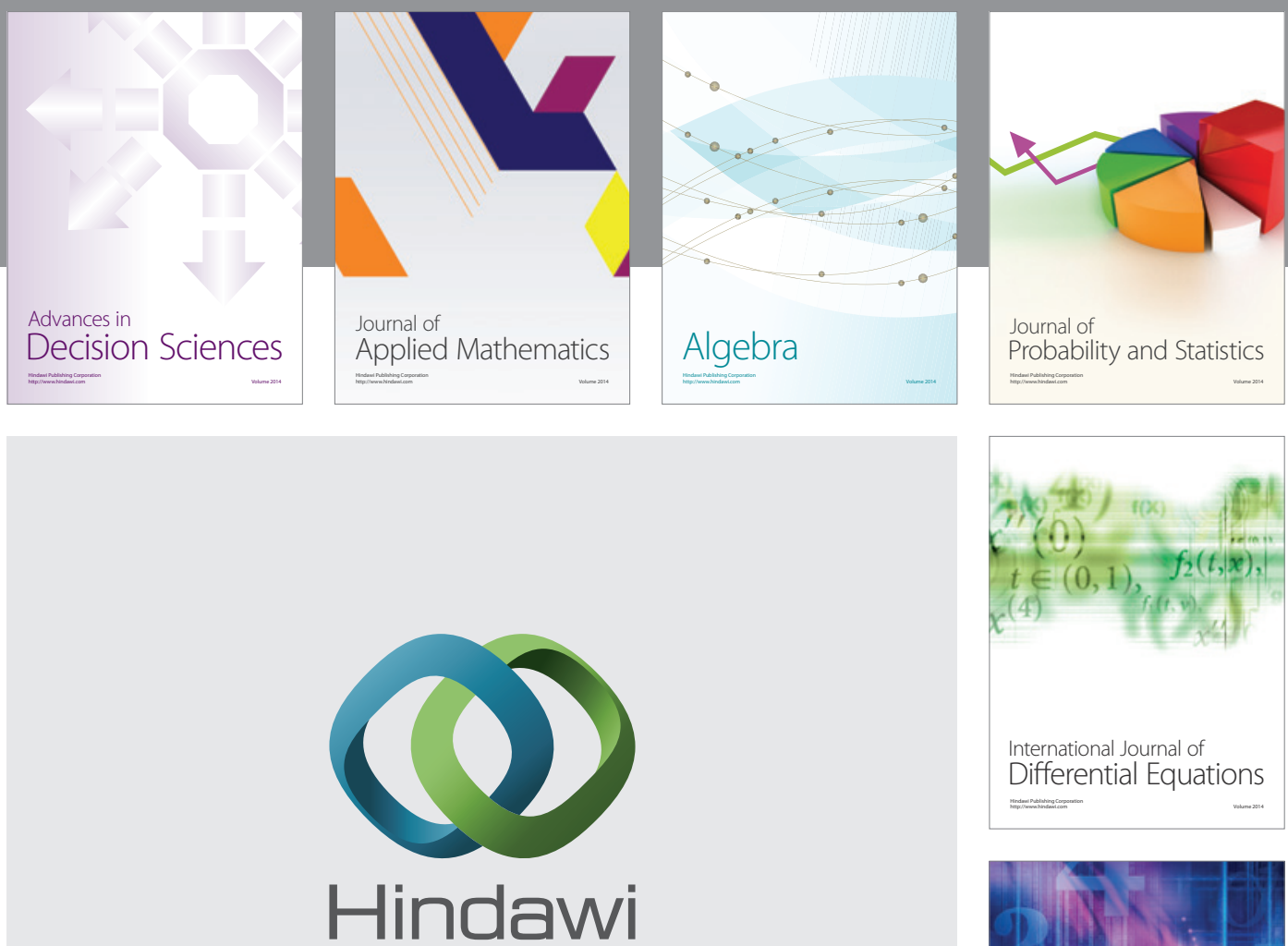

Submit your manuscripts at http://www.hindawi.com
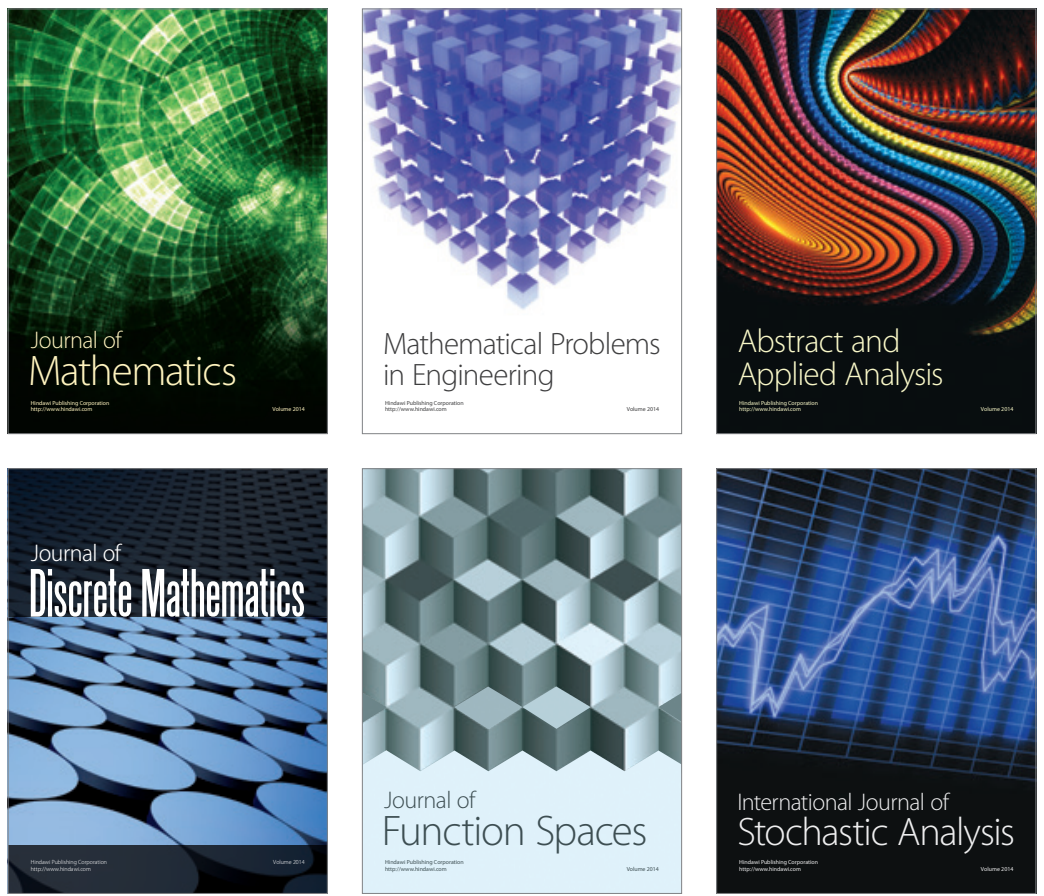

Journal of

Function Spaces

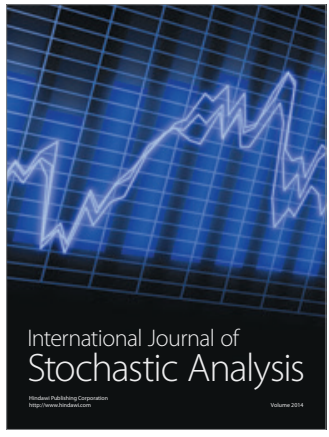

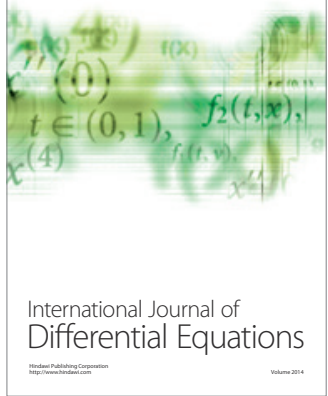
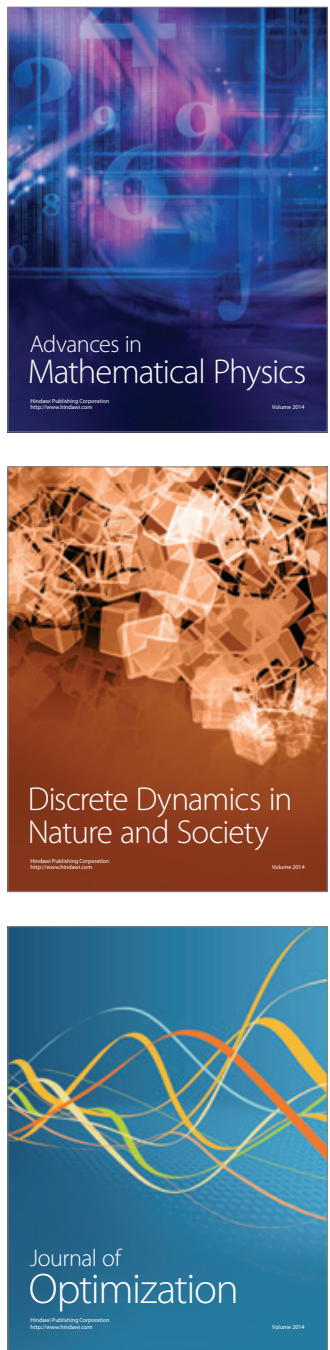